Revista Internacional de Apoyo a la Inclusión, Logopedia, Sociedad y Multiculturalidad.

Volumen 6, Número 3, diciembre 2020, ISSN: 2387-0907. DOI: https://dx.doi.org/10.17561/riai.v6.n3.2

\title{
Inclusión en el aula de Educación Física: desarrollo de la identidad de género
}

\author{
Inclusion in the Physical Education classroom: development of gender identity \\ Inklusion im Sportunterricht: Entwicklung der Geschlechtsidentität \\ Inclusion dans la classe d'éducation physique : développement de l'identité de genre \\ Inclusão na sala de aula de Educação Física: desenvolvimento da identidade de gênero \\ L'inclusione nell'aula di Educazione Fisica: sviluppo dell'identità di genere \\ Включение в класс физкультуры: развитие гендерной идентичности
}

体育の授業におけるインクルージョン : ジェンダー・アイデンティティの発達

融入体育课堂：性别认同的发展。

\author{
Adelina Merino Gutiérrez \\ Universidad de Jaén \\ amg00227@red.ujaen.es \\ Carlos García Miguélez \\ Universidad de Jaén \\ cgm00099@red.ujaen.es
}

Fecha recepción: 12/10/2020

Páginas 11-23

Fecha aceptación: 29/11/2020

\section{Resumen.}

Tal como lo reconocen los expertos en la materia, el concepto de Educación Inclusiva (EI) es complejo y aún no se encuentra definido del todo dentro de la comunidad educativa. A pesar de su creciente presencia en el entorno educativo, se observa que esta se ha mantenido ligada a la idea de que supone el avance desde el modelo de integración que favorece la atención de los niños con NEE, desconociendo su carácter más amplio y el papel que juega en el desarrollo de la identidad de género de los menores. De esta manera, se pretende establecer de forma general en qué punto se encuentra la investigación académica en el ámbito de la El en el área de Educación Física, en relación con la identidad de género. Entre otras cosas, se pudo concluir que, en la actualidad, la sociedad está claramente marcada por el binarismo hombre-mujer, masculino-femenino, a pesar de que las posibilidades de género son muy diversas. Para valorar tal diversidad es necesario abolir las normas que regulan los géneros y las expectativas hacia los individuos en función de su sexo o género.

Palabras clave: inclusión; identidad de género; educación; educación física; diversidad sexual

\section{Abstract.}

As recognized by experts, the concept of Inclusive Education (IE) is complex and it has not yet been fully defined within the educational community. Despite its growing presence in the educational environment, it is observed that it has remained linked to the idea that it implies the advance from the integration model that favors the care of 
children with SEN, ignoring its broader nature and the role that plays in the development of the gender identity of children. In this way, it is intended to establish in a general way the development of this topics in the academic research, including the area of Physical Education, in relation to gender identity. Among other things, it was possible to conclude that, at present, society is clearly marked by the binary man-woman, masculine-feminine, despite the fact that the gender possibilities are diverse. To value such diversity it is necessary to abolish the norms that regulate genders and expectations towards individuals based on their sex or gender.

Keywords: inclusion; gender identity; education; physical education; sexual diversity

\section{1.-Introducción.}

Desde que, en el año 1990, a partir de la Declaración Mundial sobre Educación para Todos, se comenzara a acuñar el modelo de Educación Inclusiva, hasta la actualidad, se han percibido muchos cambios y se han producido avances que propician el desarrollo y el asentamiento de este modelo a nivel planetario (Muntaner Guasp, Rosselló Ramón, \& de la Iglesia Mayol, 2016).

Así mismo, en los últimos años se ha convertido en uno de los elementos primordiales en la acción educativa la formación en diversidad, respetando las individualidades de cada persona. Se educa en la diversidad en ámbitos como la raza, la cultura, el sexo, las capacidades 0 aspectos físicos. Sin embargo, la diversidad en la identidad de género y en las formas de expresión de este no goza de tal privilegio (Pérez Enseñat \& Moya Malta, 2020).

Salta a la vista que el mensaje de la Educación Inclusiva ha tenido presencia con diversos matices en la mayoría de acuerdos e instrumentos legales internacionales generados, lo que ha generado que este concepto incida en las políticas y marcos normativos de muchos países, los cuales han adoptado medidas de variado calibre para favorecerla y reducir los mecanismos de los sistemas que generan exclusión, segregación o bajo desempeño (Duk \& Murillo, 2018).

A pesar de la creciente presencia de la Educación Inclusiva en el entorno educativo, se observa que esta se ha mantenido ligada a la idea de que supone el avance desde el modelo de integración que favorece la atención de los niños con NEE, desconociendo su carácter más amplio y el papel que juega en el desarrollo de la identidad de género de los menores en el entorno escolar.

Lo anterior no es diferente en el aula de Educación Física (EF) en la que persiste la idea de que la inclusión es trabajar para que los niños con NEE puedan y sean partícipes activos de las actividades que en su ámbito se desarrolla. De esta forma, con las siguientes reflexiones se quiere hacer un llamado para que este panorama comience a verse desde una perspectiva más amplia, en la que se tome conciencia de que la inclusión no se limita a las capacidades físicas del individuo, sino que abarca aspectos fundamentales como la imagen propia, la identidad, la autopercepción y la 
propia valía, aspectos que configuran una formación holística y que transcienden al sujeto corpóreo.

\section{2.-Objetivos.}

Como parte del presente ensayo académico se determinó establecer la relación existente entre el concepto de educación inclusiva y los conceptos de identidad de género - diversidad sexual. Así mismo, identificar cómo, desde el aula de Educación Física se trabaja en pro para que día a día los estudiantes de cualquier ciclo formativo accedan a una educación inclusiva real.

Adicionalmente, se pretende establecer de forma general en qué punto se encuentra la investigación académica en el ámbito de la educación inclusiva en el área de Educación Física, identificando estudios y propuestas de intervención cuyo eje temático principal se alinee con los aquí propuestos.

Se ha de tener en cuenta que el presente artículo no pretende ser una revisión exhaustiva para determinar el estado de la cuestión, sino ser un aporte a la reflexión en torno a los temas que se abordan para, eventualmente, despertar el interés entre investigadores y fomentar otros estudios en profundidad que deriven en prácticas reales en las aulas de clase.

\section{3.-Metodología.}

El desarrollo de este trabajo parte por la identificación y delimitación del tema a abordar. En primera instancia se elige el tema de inclusión educativa y se revisan diversas fuentes bibliográficas para reconocer el desarrollo de la temática en el ámbito específico de la Educación Física, identificando avances teóricos y prácticos en este campo. En segunda instancia, se revisar aportes teóricos sobre la inclusión educativa y la identidad de género para, finalmente, establecer los puntos de convergencia de los tres ejes temáticos, es decir, la inclusión en la educación física desde la perspectiva de la identidad de género.

Con lo anterior, se consultaron diversas bases de datos a través de los descriptores diversidad, género, inclusión, educación y educación física. La revisión bibliográfica se delimitó a los aportes desarrollados en los últimos 5 años (2015 - 2020), tanto en idioma castellano como en inglés, eligiendo preferentemente fuentes primarias de información, es decir, artículos de revistas científicas y libros.

\section{4.-Resultados.}

\section{1.-Educación Inclusiva.}

Tal como lo reconocen los expertos en la materia, el concepto de Educación Inclusiva es complejo y aún no se encuentra definido del todo dentro de la comunidad educativa, pues no se establece una diferencia clara entre integración escolar e inclusión 
educativa. De esta forma, se tiene que en numerosos estudios se plantea que los dos términos no significan lo mismo en todos los países. De ahí que sea preciso incidir en que, semánticamente, integrar e incluir tienen significados muy próximos, lo que deriva en que su uso sea indistinto. Sin embargo, dentro de los movimientos educativos, integración e inclusión representan filosofías y corrientes totalmente distintas.

El concepto de integración hace referencia a diferentes modelos y tendencias dentro de la misma: el centrado en el emplazamiento del alumnado; el que abarca una intervención sectorial; y el que se relaciona con la integración de tipo institucional. Es común entre estas líneas la concepción como procesos mediante los cuales los niños tienden a un fin ya establecido con anterioridad en el centro educativo.

Por su parte, la inclusión desarrolla el deseo de reestructuración y participación en el programa de la escuela, para responder a la diversidad de todo el alumnado que recibe clases. De esta forma, también es posible establecer diferentes líneas dentro de su corriente: colocación del alumnado; educación para todos y para todas; participación; y como inclusión social (Sancho Álvarez, Giner, Paula, \& Grau Vidal, 2013).

Tomando como referencia lo anterior, se puede considerar que la inclusión, en términos generales, puede ser entendida como un principio rector que orienta las políticas y prácticas educativas en todos los estudiantes y, por consiguiente, del sistema en su conjunto. No hace referencia ni se restringe a los estudiantes con necesidades educativas especiales, aunque obviamente los incluye (Duk \& Murillo, 2018). Supone que todos los alumnos se reúnan en las aulas ordinarias de las escuelas de cada comunidad, sustituyendo a la formación a tiempo completo y por horas en contextos segregados (Bunch, 2008).

La Educación Inclusiva supone un cambio global, como se ha mencionado, en el sistema educativo, con un doble objetivo: por un lado, alcanzar el éxito de todos, sin excepciones, en la Escuela; y por otro, luchar contra cualquier causa o razón de exclusión, en cualquiera de sus variantes de segregación o discriminación. El modelo se perfila como una respuesta de calidad a la diversidad existente en las aulas, desde un planteamiento equitativo, no basado en enfoques asistenciales, compensatorios 0 focalizados, sino con un carácter preventivo y global (Muntaner Guasp, Rosselló Ramón, \& de la Iglesia Mayol, 2016).

Teniendo en cuenta que la Educación Inclusiva puede ser entendida como un principio rector dentro de las políticas educativas de cualquier Estado, se considera oportuno mencionar que, derivado del Foro Europeo de Discapacidad emanó la siguiente consideración en referencia al concepto:

"La inclusión es más amplia - que la integración - ya que implica un cambio progresivo y la adaptación del sistema educativo, de tal manera que todo el mundo pueda tener sus necesidades satisfechas y prosperar" (Sancho Álvarez, Giner, Paula, \& Grau Vidal, 2013) 
Leiva \& Gómez indican que la Educación Inclusiva supone la valoración positiva de la actitud pro-comunicación y pro-interacción entre personas diferentes, enfocada en la compresión de lo diverso como factor de aprendizaje positivo y necesario en las actuales organizaciones escolares. Para estos investigadores, la Educación Inclusiva se posiciona entre el deseo de ser una propuesta educativa crítica, y la realidad de encontrarse en una encrucijada que va desde la segregación encubierta del alumnado con necesidades educativas especiales, hasta la falta de profesionales especializados, pasando por la escasa formación en Educación Inclusiva de todo el profesorado y la comunidad educativa. Reconocen que aún persiste el enfoque curricular compensatorio y deficitario en el tratamiento educativo de los menores con necesidades educativas especiales, lo que resulta cuestionable, no solo en el ámbito académico, sino por parte de los profesionales de la educación y por parte de las familias que viven con el malestar al observar que el sistema educativo no es capaz de garantizar una atención inclusiva y de calidad a sus hijos (Leiva Olivencia \& Gómez Gerdel, 2015).

Por su parte, Ainscow sugiere que en muchos países del mundo se considera a la Educación Inclusiva como un modo de atender a los niños con discapacidades dentro del contexto de la educación general (Ainscow, 2017). En esta misma línea, Cansino hace referencia a que la Educación Inclusiva

"es un modelo que se fundamenta en incluir al alumnado con Necesidades Educativas Especiales en la sesión de Educación Física, haciendo las adecuaciones necesarias no sólo del niño con NEE, sino tomando en cuenta a todos y cada uno de los integrantes del grupo para garantizar su participación" (Cansino Aguilera, 2016)

Canales et al. amplían estas consideraciones al explicar que el concepto de inclusión escolar se ha asociado a la integración de estudiantes con NEE al aula común, sin materializar cambios profundos en las prácticas y metodologías de enseñanza. Este enfoque tiene sus orígenes en Estados Unidos, en los años 60, como resultado del debate por la exclusión escolar de los menores con condiciones físicas y cognitivas diferentes (Canales Nuñez, Aravena Kenigs, Carcamo Oyarzun, Lorca Tapia, \& Martínez Salazar, 2018).

Dicha posición que puede ser extendida con la idea de Santamaría et al. quienes indican que el paradigma inclusivo ofrece atención a los alumnos con deficiencias físicas, sensoriales intelectuales que les producen dificultades en el aprendizaje, y que se posiciona como un progreso desde la perspectiva de la integración como línea de acción precedente que enmarca la intervención educativa dirigida a este grupo específico (Santamaría Fernández, Ruíz Sanchis, Puchalt, Ros Ros, \& Martín Ruíz, 2016).

De las descripciones conceptuales anteriores es posible extraer que, comúnmente, al hacer referencia a la Educación Inclusiva se establece una relación inmediata con la garantía del acceso a la educación de los menores en situación de discapacidad en un 
entorno no diferenciado. Sin embargo, tal como ya lo expresaran Duk \& Murillo en su definición, esta no debe restringirse a los estudiantes con NEE, de ahí la necesidad de hacer visible también las necesidades y preocupaciones de la comunidad LBGTI en el entorno educativo.

Esta idea toma forma y se refuerza cuando se valora la diversidad: esta conduce a respetar lo diverso, otorgándole a cada uno lo que realmente necesita, sin descuidar lo que es común a todos. Así, el sistema educativo inclusivo ha de caracterizarse por la oferta educativa de calidad para todos los alumnos, independientemente de cuales sean sus circunstancias personales - educación, género, idioma, por mencionar algunas - sociales o culturales, tomando siempre en cuenta que el campo de actuación y de vivencias del sistema han de desarrollarse en el espacio definido de la Escuela, en conjunto con las familias, el cuerpo docente y la comunidad educativa en general.

En concreto, la inclusión supone desarrollar un modelo de Escuela dirigido a la calidad, no discriminatorio, participativo en el que lo heterogéneo es un factor que enriquece (Colmenero Ruíz, 2015).

\section{2.-Identidad de género y educación.}

Tanto la Escuela como el profesorado tienen un papel crucial en la socialización del alumno, y en particular, en la construcción social del género, pues al ser los transmisores de normas y valores, influyen directamente en el imaginario social en torno al concepto de género (Pinedo González, Arroyo González, \& Berzosa Ramos, 2018).

Sexo y género son dos conceptos implementados como sinónimos frecuentemente a pesar de tener significados distintos. El primero, hace referencia a las características sexuales del individuo, pudiendo ser este masculino o femenino - hombre o mujer, respectivamente - y se fundamenta en el fenómeno biofisiológico determinado por genitales, cromosomas, gónadas, hormonas, entre otros. El segundo, aporta los rasgos psicológicos y emocionales a la persona. Es una construcción social derivada de los roles y estereotipos que determinan qué actitudes, costumbres, valores 0 características definen tanto a hombres, como a mujeres. Así mismo, la identidad de género se relaciona con el sentimiento individual del género. Dicho de otra forma, es la manera en que cada persona experimenta el género y cómo dicha vivencia aporta al sentido de la identidad, singularidad y pertenencia (Pérez Enseñat \& Moya Malta, 2020).

Abundando en lo anterior, se observa que el género se constituyó como categoría explicativa de la construcción simbólica de los hombres y mujeres tomando como referencia la diferencia sexual. Es una definición en la que se encuentra implícita la idea de que existe un tipo de relación entre ambos sexos que se produce con base al poder que ejerce el uno sobre el otro. Sin embargo, no se puede pasar por alto que en este binomio mujer-varón existe una gama de diferencias humanas que deben considerarse en el panorama educativo. Así, dichas diferencias habituales componen una sociedad diversa que obliga a aprender a vivir en el respeto a esas diferencias, 
buscando en ellas los motivos y causas para enriquecer al ser humano (Curieses, 2017).

Magallanes \& Sadoski reconocen que muchas personas suelen confundir el concepto de identidad de género, con el concepto de orientación sexual. El primero, explican, tienen que ver con el género que siente la persona, ya sea masculino, femenino, ambos o ninguno de los dos. Por otra parte, ser lesbiana, gay, heterosexual o bisexual describe quien atrae a cada persona y con quienes desea tener una relación emocional y/o sexual (Magallanes \& Sadoski, 2019).

Curises, por su parte, argumenta que la nueva perspectiva sobre la identidad de género permite identificar y valorar la pluralidad de los modos de ser y las diferencias entre los individuos, no sólo su liberación, que hasta ahora se limitaban a categorías como mujer u hombre sobre las que pesaba un referente fijo, sino que es un avance como sociedad al verse obligada a replantearse el hacer, a nivel teórico y práctico, en la política y consecuentemente en el sistema educativo, ya que esta última es también una cuestión política que está sujeta a criterios de organización e ideología social. Así se favorece y se impulsa la Educación Inclusiva coincidiendo con la necesidad de identificar y analizar contextos sociopolíticos educativos que favorezcan las prácticas inclusivas (Curieses, 2017).

Otros autores señalan que hoy en día existe una mayor apertura desde las políticas educativas en relación con las múltiples manifestaciones de la diversidad, mirada que resulta coherente con el contexto globalizado, y que ha derivado en que se tome conciencia de las identidades locales y aquellas tradicionalmente excluidas. De esta forma, los nuevos contextos legales y sociales están promoviendo una nueva forma de pensamiento, ampliando los desafíos que la heterogeneidad supone en la labor docente (Salas Guzmán \& Salas Guzmán, 2016).

En resumen, se puede reconocer que existe una clara estrategia para garantizar el derecho a la educación de todas las personas, sin embargo, son numerosas las voces que piden pensar, estudiar, proponer y comprometerse con nuevas formas de entender, enfocar y desarrollar la educación en diversidad del alumnado (Curieses, 2017).

\section{3.-Educación Inclusiva en el aula de Educación física.}

Para hablar de Educación Inclusiva en el aula de EF es necesario regresar un poco en el tiempo para reconocer que en los últimos años se ha pasado del paradigma de la integración, donde los alumnos con o sin discapacidad coincidían en un mismo centro educativo, recibiendo formación a partir de un currículo diferenciado para atender las necesidades específicas; al paradigma de la inclusión, donde las diferencias se toman como una categoría inherente al hecho educativo y que conduce a que todos los alumnos sean formados a partir de un mismo currículo. 
Sin embargo, esta idea dista mucho de la realidad en la medida en que en el área de EF se requiere realizar adaptaciones del currículo para atender tal diversidad de capacidades (Santamaría Fernández, Ruíz Sanchis, Puchalt, Ros Ros, \& Martín Ruíz, 2016).

Partiendo de la idea de que la Educación Inclusiva es una cuestión de derechos, tal como lo señala Muntaner et al., en donde todos los alumnos de una comunidad aprenden juntos, indistintamente de sus condiciones personales, sociales o culturales. Con ella, se pretende lograr una escuela que se adapte a las características de los alumnos y no a la inversa.

Este modelo se ha de entender como un proceso de búsqueda que cada comunidad educativa debe llevar a cabo, para encontrar formas mejores y más eficaces para responder a la diversidad del alumnado. Así mismo, como un proceso de mejora y desarrollo institucional que compromete a toda la comunidad escolar (Muntaner Guasp, Rosselló Ramón, \& de la Iglesia Mayol, 2016).

Cansino matiza que es necesario comprender cómo se identifica el deporte en la sociedad, por lo que propone el siguiente esquema:

Ilustración 1.-Identificación social del deporte, diferenciación entre el deporte adaptado $y$ el inclusivo.

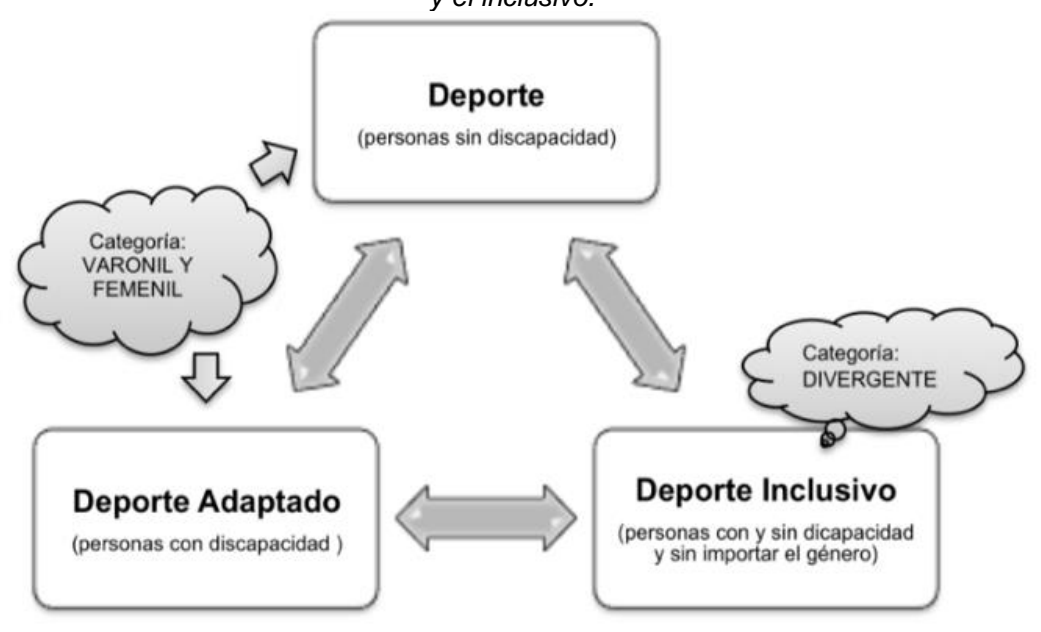

Fuente: (Cansino Aguilera, 2016)

De acuerdo con esto, se observa que paulatinamente los profesionales de la EF se abren a nuevas acepciones del concepto de Educación Inclusiva, reconociendo que esta trasciende la discapacidad y resulta más amplia, abarcando temas como el género (Cansino Aguilera, 2016). 


\section{4.-Desarrollo de la identidad de género en la Educación Física inclusiva.}

No resulta ajeno para nadie que en España las situaciones de discriminación hacia el colectivo LGTBI no son hechos aislados, situación que en el deporte parece no ser diferente. En este contexto, el colectivo se ha convertido en foco de marginación, aversión y opresión, viéndose forzado a ocultar aspectos de su propia identidad sexual y de género por temor a convertirse en objeto de intimidación, rechazo, violencia, desprecio o aislamiento. Algunos estudios indican que estos comportamientos no se limitan a la esfera deportiva, sino que trasciende de manera visible al área de EF (Ramírez Díaz \& Cabeza Ruíz, 2020).

Tal como lo reconocen Pérez \& Moya, la Escuela es un espacio donde se reproduce constantemente la cultura heteronormativa y de patrones, roles y normas de género, en particular desde el aula de EF, en la que el género suele ser una característica tenida en cuenta a diario. De esta forma, las expectativas docentes hacia el alumnado varían en función del género, desconociendo que las distinciones reproducen las desigualdades y las estigmatiza.

Valorando la situación desde la perspectiva del alumnado, se evidencia que la asimilación de los estereotipos tiene altas probabilidades de derivar en discriminación y acoso hacia aquellos que traspasan los límites sociales del género (Pérez Enseñat \& Moya Malta, 2020).

Evidencia de ello es que la discriminación y exclusión por homofobia y transfobia es una constante en la sociedad actual, lo que se está reproduciendo en mayor o menor medida en los centros educativos:

"ser o parecer lesbiana, gay, bisexual o trans y no cumplir con las normas de género asignadas culturalmente a hombres y mujeres son algunas de las causas de acoso escolar: motivan desde el insulto y la burla, hasta la exclusión y la violencia física" (Pichardo Galán, de Stéfano Barbero, Sáenz, \& Williams Ramos, 2015)

En concordancia con lo anterior, se puede comentar que el aula de EF destaca como un espacio que ofrece oportunidades de apoyo, colaboración y desarrollo de conductas prosociales en el alumnado. A su vez, se presenta como una herramienta significativa para lograr los objetivos de un proyecto inclusivo, ya que la acción educativa asociada a la actividad física promociona la interacción social y el desarrollo de valores inherentes al deporte, pues esta posee como valor intrínseco su capacidad para generar espacios igualitarios en los que se favorecen las prácticas inclusivas (Canales Nuñez, Aravena Kenigs, Carcamo Oyarzun, Lorca Tapia, \& Martínez Salazar, 2018).

Con esto, y a manera de cierre, se aportan a continuación una serie de buenas prácticas a (Salas Guzmán \& Salas Guzmán, 2016) implementar en el entorno del aula de $E F$, de forma tal que se dé un paso en dirección hacia la inclusión real y efectiva: 
- La diversidad es una marca humana. Respecto al género y la sexualidad los docentes tienden a uniformar a niños y niñas con los mismos moldes normalizados. Una parte básica de la Educación Inclusiva es deconstruir los binarios sexuales y de género.

- Las (Homo-Bi) sexualidades son esencias construidas, es decir, la identidad sexual se estructura en relación con un contexto socio-cultural específico. Para apoyar a las personas LGBTI es mejor considerar los factores que contribuyen a la homo/lesbo/bifobia y el heterosexismo, en lugar de enfocarse en las causas de su homosexualidad, lesbiandad y/o bisexualidad.

- La homofobia y el heterosexismo son adquiridos, es decir, el odio y el medo a las personas que aman a otras personas de su mismo género se adquieren tempranamente. El cuerpo docente debe confrontar los prejuicios personales con los que se ha adoctrinado a lo largo de décadas de heterosocialización.

- La inocencia infantil es una ficción absoluta. Los adultos disfrazan su temor a la incomodidad, subestimando la capacidad de los menores para pensar de manera crítica y comprender en profundidad su entorno.

- La familia es lo primero. Los conceptos de parentalidad y familia se han transformado, por lo que la importancia se traslada a que los educadores identifiquen y enseñen sobre las nuevas realidades, por ejemplo, de que algunas familias tienen dos madres 0 dos padres que se aman.

\section{5.-Conclusiones.}

Coincidiendo con lo expresado por Duk \& Murillo, la revisión bibliográfica permitió establecer que no es sorprendente escuchar hablar del sistema y la Escuela inclusiva, de políticas, gestión o prácticas inclusivas, de currículo inclusivo, hasta de profesor inclusivo. Se observa que el enfoque inclusivo en la perspectiva educativa se ha instalado ampliamente, lo que sin duda resulta positivo. Sin embargo, esta misma situación advierte del riesgo de que el uso indiscriminado del término derive en la aplicación de una etiqueta más, carente de contenido y que conduzca a la pérdida de significado o culmine considerándose una moda educativa más. Estos hechos conducen a reconocer que lo que fundamenta a lo inclusivo es la demanda de acceso igualitario a las oportunidades educativas de calidad, para todos los estudiantes, basada en el reconocimiento de las diferencias, lo que se traduce en una educación más equitativa (Duk \& Murillo, 2018).

Tomando a la Educación Inclusiva como el paradigma que ha desencadenado debates en múltiples lugares del mundo, se puede afirmar que esta requiere la identificación y eliminación de todo tipo de barreras, incluidas las actitudinales, bien sea a nivel de políticas educativas, por profesionales de la docencia o sociedad en general como consecuencia de culturas y creencias alejadas del paradigma en cuestión (Leiva Olivencia \& Gómez Gerdel, 2015).

Lo anterior supone poner énfasis en aquellos grupos de alumnos que podrían estar en riesgo de marginalización o exclusión por cuestiones de orientación sexual y/o 
identidad de género, lo que implica darles voz y tomarles en consideración, tal y como es el propósito de la presente reflexión.

Se ha de superar la Educación Inclusiva vista como una simple cuestión organizativa, curricular y/o metodológica; para avanzar en su compresión como una forma distinta de entender y practicar la educación, que propone y exige metodologías didácticas, propuestas curriculares y modelos organizativos donde puedan aprender juntos alumnos diferentes.

Para ello, es necesario fundamentarse en tres principios: aprender a trabajar en los centros con la diversidad del alumnado, mantener la igualdad de oportunidades y mantener las altas expectativas de éxito en el conjunto de estudiantes, lo cual, indiscutiblemente, se perfila como grandes retos del modelo educativo actual (Muntaner Guasp, Rosselló Ramón, \& de la Iglesia Mayol, 2016).

Se puede considerar, entonces que, el modelo de Educación Inclusiva es válido para una sociedad democrática, que, en principio, asume las diferencias y las valora además de tenerlas en cuenta para que las personas que, en un grupo determinado, se singularizan más por sus características particulares, dispongan de las mismas oportunidades de educación, formación y desarrollo que el resto. Así, se reconoce que este tipo de educación implica que todos los alumnos de una determinada comunidad aprendan juntos, independientemente de sus condiciones personales, sociales y/o culturales, oponiéndose a cualquier tipo de segregación y a cualquier argumento que fomente la separación o pretexto en el ejercicio de los derechos a la educación (Colmenero Ruíz, 2015).

En esta línea, avanzar hacia una educación inclusiva se perfila como un desafío importante para el profesorado de Educación Física, pues en su conjunto, debe superar la limitación al desarrollo de las actitudes físicas que implican la asignatura, para transformar el aula en un espacio de aprendizaje social acorde con los intereses y características del alumnado (Canales Nuñez, Aravena Kenigs, Carcamo Oyarzun, Lorca Tapia, \& Martínez Salazar, 2018).

En relación con el concepto de género, se puede comentar que, en la actualidad, la sociedad está claramente marcada por el binarismo hombre-mujer, masculinofemenino, a pesar de que las posibilidades de género son muy diversas. Para valorar tal diversidad es necesario abolir las normas que regulan los géneros y las expectativas hacia los individuos en función de su sexo o género. Específicamente al entrar en el ámbito de la EF se observa que los resultados esperados del alumno se relacionan fuertemente con el género, así como las exigencias y baremos, incidiendo en los contenidos donde se diferencian los desarrollados para chicos y para chicas. De igual manera, la EF es una asignatura en la que se hace uso específico de instalaciones marcadas por el género, como lo son vestuarios y baños. Lo anterior deriva en que el aula de EF y sus espacios complementarios sean lugares en los que continuamente el niño deba definir y gestionar su identidad de género (Pérez Enseñat \& Moya Malta, 2020). 
Trasladando las reflexiones de otros autores, se considera oportuno comentar que la en el ámbito educativo se sigue construyendo a las personas LGTBI mediante identidades irreductibles y desde una lógica de atención a las minorías, donde las diferencias con el grupo mayoritario heterosexual son invisibilizadas. En la construcción de la otredad irreductible de este colectivo, se evidencia un doble mecanismo: por un lado, la diferenciación radical, y por otro, el establecimiento de categorizaciones fijas. Esto deriva en una operación de diferenciación jerarquizada en la que la figura de la diversidad sexual se concibe como diferente respecto a una supuesta comunidad de iguales - compuesta por personas heterosexuales - y en relación asimétrica respecto a los miembros del grupo mayoritario. De esta forma, se está ante un fenómeno de subalternización que conlleva que los sujetos sean posicionados marginalmente al sistema, se conceptualicen y representen desde el discurso de la otredad, desde la posición hegemónica. Así, los dispositivos de intervención educativa continúan reproduciendo los discursos que consideran a estos sujetos como carentes o deficitarios, limitando sus posibilidades (Galaz, Troncoso, \& Morrison, 2016).

En resumen, con el desarrollo de la presente reflexión se pudo establecer la relación existente entre la educación inclusiva, la identidad de género y el aula de EF, siendo esta última el espacio idóneo para desarrollar las acciones educativas que se fundamentan en el paradigma inclusivo y que trasciende la barrera de la educación para niños con NEE, entendiendo que su centro de atención es la diversidad, y que por ende, da soporte al desarrollo psicológico, social y no únicamente deportivo del alumnado.

Así mismo, se pudo determinar que la Educación Inclusiva como línea de investigación académica cuenta con un cuerpo teórico consolidado y extenso que, sin embargo, no ha permitido establecer con claridad sus fundamentos, los cuales irán variando en función del momento histórico y autor que los relacione. En cuanto a su análisis desde la perspectiva de la EF se observa que es común relacionarla con la formación de niños con NEE, evidenciando la persistencia de la concepción de la EF como asignatura limitada al desarrollo de actitudes físicas y desconociendo la integralidad del ser humano.

Finalmente, se espera que con las ideas presentadas a lo largo de esta reflexión sean más los investigadores y académicos que se interesen por abordar los temas de diversidad, identidad sexual e inclusión en el ámbito de la EF pues se pudo identificar que los trabajos en estos campos son escasos y existe una necesidad real de dar voz a aquellos que por su condición están en riesgo de exclusión.

\section{6.-Referencias.}

Ainscow, M. (2017). Haciendo que las escuelas sean más inclusivas: lecciones a partir del análisis de la investigación internacional. Revista Educación Inclusiva, 5(1), 39-49. 
Bunch, G. (2008). Claves para una educación inclusiva exitosa. Una mirada desde la experiencia práctica. Revista Educación Inclusiva(1), 77-89.

Canales Nuñez, P., Aravena Kenigs, O., Carcamo Oyarzun, J., Lorca Tapia, J., \& Martínez Salazar, C. (2018). Prácticas pedagógicas que favorecen u obstaculizan la inclusión educativa en el aula de educación física desde la perspectiva del alumnado y profesorado. Retos(34), 212-217. https://doi.org/10.47197/retos.v0i34.59620

Curieses, P. (2017). Por una escuela inclusiva. Las fronteras del género. Revista Internacional de Educación para la Justicia Social (RIEJS), 6(2), 63-79. https://doi.org/10.15366/riejs2017.6.2.004

Duk, C., \& Murillo, J. (2018). El mensaje de la Educación Inclusiva es simple, pero su puesta en práctica es compleja. Revista Latinoamericana de Educación Inclusiva, 12(1), 11-13. https://doi.org/10.4067/S0718-73782018000100011

Leiva Olivencia, J. J., \& Gómez Gerdel, M. d. (2015). La educación inclusiva como constructo pedagógico en el alumnado universitario de educación primaria. Revista nacional e internacional de educación inclusiva, 8(2), 185-200.

Muntaner Guasp, J. J., Rosselló Ramón, M. R., \& de la Iglesia Mayol, B. (2016). Buenas prácticas en educación inclusiva. Educatio Siglo XXI, 34(1), 31-50. https://doi.org/10.6018/j/252521

Pinedo González, R., Arroyo González, M. J., \& Berzosa Ramos, I. (2018). Género y educación: detección de situaciones de desigualdad de género en contextos educativos. Contextos $\quad$ Educativos(21), 35-51. https://doi.org/10.18172/con.3306

Ramírez Díaz, A., \& Cabeza Ruíz, R. (2020). Actitudes hacia la diversidad sexual en el deporte en estudiantes de educación secundaria. Retos(38), 654-660. https://doi.org/10.47197/retos.v38i38.77934

Santamaría Fernández, R., Ruíz Sanchis, L., Puchalt, J. M., Ros Ros, C., \& Martín Ruíz, J. (2016). Inclusión en las aulas de Educación Física. Estudio de casos. Sportis. Revista técnico-científica del deporte escolar, educación física y psicomotricidad, II(3),

496-514. https://doi.org/10.17979/sportis.2016.2.3.1511 